\title{
Las crisis del Habitar: Cine y publicidad en el Branded Content Cinergía - Gas Natural Fenosa
}

Aarón Rodríguez Serranoํㅣ | serranoa@uji.es

Samuel Gil Soldevilla² I ssoldevi@uji.es

Javier Marzal Felici ${ }^{3}$ | marzal@uji.es

UNIVERSIDAD JAUME I

Resumen: El presente trabajo propone el análisis textual de los cuatro cortometrajes que conforman la campaña de Branded Content Cinergía (Paco Plaza, Rodrigo Cortés, Jaume Balagueró y Juan Cruz, 2015), generada para la compañía Gas Natural Fenosa. En dicho análisis, observamos cómo las distintas piezas utilizan los recursos audiovisuales habituales del género de terror para realizar una lectura crítica del habitar en el contexto de la actual crisis económica e ideológica. Para ello, en primer lugar explicitaremos la importancia del objeto de estudio, contextualizándolo después en la propia trayectoria publicitaria de Gas Natural - Fenosa. Estudiaremos las relaciones entre cine, energía y modos de habitar, tomando brevemente como antecedente el espot televisivo Happiness (Huigo Menduña, GNF, 2010). Una vez clarificados los antecedentes, mostraremos cómo en los cuatro cortometrajes se encuentran notables divergencias entre los objetivos y el posicionamiento de la marca y el propio contenido ficcional, tanto desde una perspectiva de forma fílmica como desde una perspectiva estrictamente narrativa. Para ello, analizaremos los cortometrajes en torno a tres parámetros distintos en los que se manifiestan los problemas del habitar contemporáneo: el marco de lo doméstico, el marco de la identidad subjetiva, y finalmente, el marco de la propia creación artística.

Palabras clave: Branded Content - Cinergía - Gas Natural Fenosa - Análisis fílmico - Publicidad

Abstract: Our work will develop a textual analysis of the short movies included on the Branded Content strategy "Cinergía" (Paco Plaza, Rodrigo Cortés, Jaume Balagueró and Juan Cruz, 2015), developed for the Spanish energetic company Gas Natural Fenosa. In our analysis, we will see how the different stories are built over the classical resources of the horror film as a reflection of the "dwelling" (as a heideggeran concept) in the context of the spanish economical and ideological crises. To achive our goal, we will start proposing a context for the object of our study, starting with the television spot "Happiness" (Huigo Menduña, GNF, 2010). After that, we will show how the four short movies shown different gaps between the inner philosophy of the brand and the morbid textual materials used during the filmation of each piece. We will focus our analyse over three concrete concepts: the contemporary problems of dwelling, the marks of the subjective crisis and, of course, the limits of the artistic manifiestation.

Key words: Branded Content - Cinergía - Gas Natural Fenosa - Film Analysis - Advertising

\footnotetext{
${ }^{1}$ Doctor en Ciencias de la Comunicación (UEM), licenciado en Comunicación Audiovisual (UEM) y en Filosofía (UNED). Personal Investigador Contratado Doctor en la Universitat Jaume I, imparte las asignaturas Narrativa Audiovisual y Cultura Visual y de medios de comunicación de masas.

2 Licenciado en Publicidad y Relaciones Públicas (Universitat Jaume I), miembro del grupo de investigación ITACA-UJI, actualmente es personal investigador en formación y se encuentra desarrollando su tesis doctoral en el ámbito de la publicidad y sus relaciones con la teología.

${ }^{3}$ Catedrático de Comunicación Audiovisual, Director del Departamento de Ciencias de la Comunicación de la Universitat Jaume I. Licenciado en Comunicación Audiovisual, Filología Hispánica y Filosofía y Ciencias de la Educación por la Universidad de Valencia, y máster en Comunicación y Educación por la Universidad Autónoma de Barcelona. Es miembro de la Asociación Española de Historiadores del Cine (AEHC), de la Asociación Española para la Investigación en Comunicación (AE-IC), de la Asociación para el Desarrollo de la Comunicación (adComunica) y de DirCom, Asociación de Directores de Comunicación.
} 


\section{Introducción y método}

El último trimestre de 2014 se anunció públicamente la primera edición del Proyecto Cinergía, desarrollado al alimón por la compañía energética Gas Natural Fenosa y el grupo de comunicación Atresmedia. Se trataba de un conjunto de cuatro cortometrajes dirigidos por prestigiosos realizadores españoles (Jaume Balagueró, Paco Plaza, Rodrigo Cortés y Juan Cruz) orientados hacia la concienciación de diversos aspectos relacionados con el consumo sostenible de energía en los hogares. Destinados a un público adulto y familiarizado con una serie de huellas genéricas -temáticas y formales- directamente conectadas con el cine de terror, fueron exhibidas tanto en festivales especializados (San Sebastián, Sitges, Madrid Premiere Week...), en diferentes emisiones televisivas durante los primeros meses de 2015, y finalmente, a través de Youtube y de la propia página web de la compañía energética. Se cubría así todo el espectro de difusión multipantalla, asegurándose que los diferentes sectores poblacionales, según sus hábitos de consumo audiovisual (Cha, 2013), podían acceder a los contenidos.

Los cuatro cortometrajes forman, sin duda, un ejemplo inusual de Branded content tanto por el alto nivel creativo de sus materiales como por las interesantes conclusiones que, como veremos, arroja su análisis. A diferencia de otros productos similares que se apoyan directamente en estrategias relacionadas con el product placement tradicional (Soba \& Aydin, 2013), es necesario hacer notar que la marca apenas se manifiesta en los operadores visuales de la enunciación. De hecho, su presencia se limita a un logotipo que abre y cierra el discurso, y a una breve aparición en el corto de Jaume Balagueró, con forma de tarjeta de presentación del protagonista.

La marca, por su propia posición dentro de la enunciación, queda a la vez desgajada del espacio del relato, pero también ocupando el espacio mismo de la su apertura (González Requena, 2006: 10). Emergiendo de un profundo fondo negro, su logotipo corresponde en la cadena simbólica publicitaria a la huella tradicional de la productora. Y al igual que ésta, se apropiará de todo un utillaje de entretenimiento para generar efectos sobre lo real. Resonancias que repercuten más allá de lo que los intereses de la propia marca podrían recomendar y que, como veremos, esconden un profundo temblor económico e ideológico (González Sánchez y Gutiérrez San Miguel, 2013).

El objetivo de nuestro presente trabajo es, por lo tanto, preguntarnos por la manera en la que una marca como Gas Natural - Fenosa ha propuesto, en los cuatro cortometrajes que 
configuran la primera temporada de Cinergía una forma audiovisual (Zumalde, 2011) publicitaria y cinematográfica en la que se anuda, con toda precisión, ese problema capital de nuestro tiempo que es el del habitar, tanto en su dimensión sociológico-filosófica como en su dimensión estrictamente económica. Exploración no exenta de riesgos e incluso contradicciones que emergen, de manera irremediable, en el momento en el que el Branded content requiere del arte $-y$ de los códigos de los géneros narrativos audiovisuales tradicionales- para levantar efectos de significación.

\section{Desarrollo}

\subsection{Antecedentes empresariales: Gas Natural, el cine y el problema del habitar}

En cualquier caso, y para ofrecer una primera justificación sobre la importancia que el concepto mismo del habitar ha tenido en el trayecto de Gas Natural - Fenosa, merece la pena recordar brevemente alguno de sus antecedentes. La conexión entre la marca y el mundo del cine se había venido desarrollando desde 2011 en distintas colaboraciones que incluyen la participación en los principales festivales de cine de nuestro país -Sitges, San Sebastián, Málaga...-, así como la subvención parcial de distintas salas de cine de la distribuidora Cinesa.

Durante sus primeros años de apoyo, la marca destinó sus estrategias exclusivamente en los campos de la distribución y la exhibición cinematográfica. Su línea de trabajo se desarrollaba atravesada por una idea: generar una conexión entre el espacio doméstico y la sala de cine. Conexión en la que el concepto mismo de habitar no aparecía problematizado, dando por sentada la existencia de una categoría espacial "pura" vinculada con la comodidad, el descanso y el ocio. Así, pudimos asistir al montaje en diversos festivales de instalaciones como un "sofá inmensamente cómodo" en la Madrid Premiere Week de 2011 o los autodenominados "pantunflas tour" que pretendían hacer que los participantes se sintieran "como en casa"... -, de tal manera que su posición como marca quedaba indefectiblemente vinculada a lo hogareño, lo íntimo, lo doméstico.

Del mismo modo, las acciones estáticas en cadenas de exhibición se desarrollaban tanto en salas eminentemente familiares y destinadas al consumo masivo -la cadena Cinesa, como es sabido (Sabatés, 2012), basa su notoriedad en su inserción en centros comerciales y otros espacios de consumo netamente postmodernos (Friedberg, 1993)-, como en los Festivales entendidos simbólicamente en tanto reuniones de unión de la comunidad cinéfila. Los 
primeros tenían la ventaja de acercar directamente la marca a los pequeños consumidores potenciales, y los segundos ejercían una indudable labor como escaparate mediático en todo tipo de medios de comunicación.

En estas primeras acciones, la marca había posicionado sus esfuerzos en entenderse desde la etiqueta de la cercanía, de la familiaridad, de lo íntimo. La construcción simbólica de la propuesta parece generar una conexión inconsciente entre las fuerzas de cohesión entre los individuos y las fuerzas energéticas proporcionadas por la marca que garantizaban el correcto funcionamiento en el hogar. En el siguiente epígrafe tendremos la ocasión de justificar esta idea con un estudio de caso concreto.

Hasta 2014, las campañas cinéfilas se centraban en la constante referencia a los espacios domésticos -la cocina, la sala de estar...- mediante una suerte de guiños "arquitectónicopublicitarios" (Colomina, 2010). Se señalaba una suerte de complicidad entre las domesticidades de los individuos, el consumo cinematográfico y la propia presencia de la marca como un elemento clave en el correcto desarrollo del programa ideológico del espacio íntimo. Así quedaba marcado, por ejemplo, en el primer eslogan de la campaña: "Al cine, como en casa". La sala cinematográfica se comprendía entonces como una extensión natural del hogar, reconocible, rubricada con precisión por las fotografías promocionales que acompañaban siempre en las marquesinas y los materiales de prensa: familias de clase alta, jóvenes, blancas, sonrientes y con varios hijos. La casa/cine se convertía para la marca en un espacio de integración familiar -técnica, por lo demás, habitual en publicidad (Arconada Melero, 1998)- en el que se generaba una evidente exclusión icónica de los Otros espectadores de cine -los solteros, los ancianos, los homosexuales o los emigrantes-, del mismo modo que la programación en las salas patrocinadas coincidía con una exclusión flagrante de los llamados "Otros cines" o "cines periféricos" (Elena, 1999).

\subsection{Antecedentes publicitarios: Happiness (Huigo Menduña, GNF)}

Siguiendo a Freud, podríamos señalar que la idea que latía detrás de las políticas del programa Cine Gas Natural - Fenosa era la de generar una suerte de experiencia a propósito de lo Heimlich, lo familiar. Cine y marca querían ser entendidos como aquello que es "perteneciente a la casa, no ajeno, familiar, doméstico, de confianza e íntimo" (Freud, 1979: 222). Como señalábamos anteriormente, la conexión entre energía creativa y humana y energía ofrecida por la marca está literalmente escrita en su publicidad televisiva. Así, por 
ejemplo, en el spot Happiness -utilizado además para anunciar la nueva marca tras la fusión entre Gas Natural y Unión Fenosa en 2010-, se desarrollaban una serie de conductas ejemplificadoras protagonizadas por una pareja de jóvenes a los que se les presuponía su buena voluntad gracias a la energía de su hogar.

Happiness estaba compuesto por un total de 33 planos que se reproducían temporalmente invertidos, de tal manera que la jornada laboral transcurría al revés: el anuncio terminaba con el despertar de la protagonista. Valiéndose de una técnica cinematográfica que se había ensayado con toda su brutalidad filosófica explícita en películas como Irreversible (Irréversible, Gaspar Noé, 2002) o Las reglas del juego (The rules of attraction, Roger Avary, 2002), la inversión del tiempo fílmico al pasar por el tamiz de lo publicitario suturaba, en primer lugar, el pánico que podría emerger al afrontar con toda seriedad la violencia y la angustia que acompañan al paso del tiempo.

Las imágenes "invertidas" mostraban, por su parte, únicamente una nómina de buenas acciones protagonizadas por una joven pareja y destinadas a salvaguardar de pequeñas catástrofes cotidianas a ancianos, niños y compañeros de trabajo. Toda la fotografía del espot se construía en tonos cálidos, y el montaje (salvo una leve aceleración al final), disponía de manera armónica los cambios de plano, incluyendo algunos zooms sobre el eje óptico.

En aquella ocasión, el logotipo de la marca se posicionaba al final -es decir, en el espacio de lo doméstico, del despertar, del amable desayuno entre los enamorados, el espacio de lo hogareño, y, al contrario que en Cinergía, sobre un radiante fondo blanco.

La propia voice over rubricaba, al final del anuncio: "Porque sabemos dónde se genera la felicidad, hemos unido el gas y la electricidad para conseguir el máximo bienestar dentro de tu casa. Y así, quizás, conseguir un poco más de bienestar fuera".

Con lo que, finalmente, hemos llegado al núcleo mismo del problema: la propia percepción popular de las empresas energéticas en el complejo tablero de la crisis económica. La solución ofrecida por el propio anuncio es desarmante: la conexión entre hogar y felicidad mediante la negación del paso de tiempo engancha con una suerte de "buen deseo" esbozado en ese dubitativo quizás que pronuncia la voz en off- que pone en manos de sus (aparentemente alegres) consumidores un mecanismo social que se despliega por sí mismo. Es el individuo el que, con sus pequeños gestos, ayuda a los grandes colectivos menos favorecidos. 
La empresa, por su parte, queda completamente excluida de su responsabilidad en los posibles desajustes económicos y sociológicos: no es culpable de participar de alguna manera en unas políticas de dirección injusta, en un mal reparto de los salarios o incluso en la aceptación explícita de políticas de lo que Slavoj Zizek llamó "violencia sistémica" (2009: 10) contra la ciudadanía. No está de más recordar que, según un informe publicado por El Economista con fecha del 10 de Mayo de 2015, los beneficios de Gas Natural Fenosa alcanzaron los 404 millones en el primer trimestre del presente año, esto es, un 0,7\% más que en el primer trimestre del año anterior. Estos buenos datos contrastan con la incómoda e incomprensible política de dichas empresas y del Gobierno de España de paralizar la entrada de otras formas más económicas, ecológicas y responsables de suministro energético y renovable.

En Happiness, al contrario, el motor de cambio social no pasa por la empresa, sino por la conducta responsable y humana de sus consumidores, que potenciados por esa desconocida, inaprehensible e hipotética "energía hogareña", pueden suturar el tejido social desgarrado por las malas gestiones económicas con una renovada vocación de servicio.

Sin duda resulta paradójico, como lo es también toda la estrategia de comunicación que analizaremos en las siguientes páginas a propósito de Cinergía: bajo la justificación ideológica de generar mecanismos de formación para la ciudadanía a propósito del consumo responsable y la eficiencia energética -estrategias que, como ya hemos señalado, la propia compañía no contempla ante la perspectiva de perder cuota de mercado a favor de las energías renovables-, la marca ha caído de manera irremediable en los terrenos del Unheimlich, al menos en dos aspectos.

En el primero, el más urgente, las eléctricas han demostrado que no son nada hogareñas ni familiares en tanto se niegan a que nosotros, en nuestra propia casa, podamos acceder a una energía mucho más asequible, sana para el medio ambiente y buena para nuestros bolsillos. En el segundo, porque sus planteamientos audiovisuales han mostrado ya a las claras que la fantasía del "habitar perfecto" al que se aferraban en sus campañas anteriores resultaba, en pleno 2015, insostenible textualmente.

\subsection{Cinergía y los espacios de horror}

Basta con ofrecer algunos datos rápidos para entender hasta qué punto nos encontramos lejos de las ideas a propósito del "hogar feliz" desarrollados por la marca en sus estrategias 
publicitarias de hace apenas cuatro años. Tres de los cortometrajes terminan con sus protagonistas brutalmente asesinados o condenados a un sufrimiento eterno. Dos de ellos plantean explícitamente la casa como un espacio agresivo, fantasmal, un espacio literalmente in-habitable. En los otros dos, son espacios peligrosamente cercanos a los nódulos simbólicos habituales de habitabilidad los que llevan a la muerte: el coche particular o el puesto de trabajo (vinculado, además, al mundo del arte). Tres de ellos están protagonizados por personajes desoladoramente solitarios y sin ningún vínculo afectivo evidente. Parecería, por tanto, que nos encontramos ante un interesante dilema comunicativo. Por un lado, el mensaje que Gas Natural Fenosa pretende proporcionar a su audiencia está claramente alineado con los intereses que conectan su particular responsabilidad social corporativa (centrados en el medio ambiente, la eficiencia energética y el consumo responsable), así como con la propia voluntad de prestigiarse frente a sus consumidores. Por otro lado, la propia nómina de directores elegidos para la primera hornada -tres de ellos especializados específicamente en el cine de terror (Olivares Merino, 2011) o en el thriller- garantiza una buena nómina de mensajes distópicos, sádicos, muchos de ellos diametralmente opuestos a la trayectoria publicitaria anterior de la propia marca.

\subsubsection{Habitar (lo real de) lo doméstico}

El problema del habitar se encuentra, sin duda alguna, en el centro mismo de las relaciones entre cine, sujeto y pensamiento en el siglo XX. Es necesario recordar la definición de la angustia como rasgo constitutivo del sujeto ya propuesta por Martin Heidegger en la primera parte de Ser y tiempo. Rasgo que, además, está directamente vinculado con un no-tenercasa, un no-poder-habitar: la angustia es el "no-estar-en-casa ["Zuhause-sein"] (...) El noestar-en-casa debe ser concebido ontológico-existencialmente como el fenómeno más originario" (Heidegger, 2009: 207-208). Idea que se repetirá, aunque con matices, en su célebre Construir, habitar, pensar (Heidegger, 1994: 127-142). De este último texto, lo que nos interesa especialmente aquí es la particular conexión que el filósofo alemán establece entre el habitar y la muerte. Entre los distintos rasgos que conforman el "buen habitar", uno de ellos tiene que ver necesariamente con la doble incorporación de lo sagrado como elemento simbólico mayor de la casa familiar, y a la vez, la aceptación de la mortalidad de los seres humanos: 
Los mortales habitan en la medida en que conducen su esencia propia -ser capaces de la muerte como muerte- al uso de esta capacidad, para que sea una buena muerte. Conducir a los mortales a la esencia de la muerte no significa en absoluto poner como meta la muerte en tanto que nada vacía; tampoco quiere decir ensombrecer el habitar con una mirada ciega dirigida fijamente al fin (1994: 132).

Los dos cortometrajes del proyecto Cinergía directamente relacionados con el problema de la habitabilidad de los espacios domésticos (Inquilinos y Domonic) hacen hincapié precisamente en esta incapacidad del habitar para conducir a una buena muerte. Antes bien, en el primero la casa se convierte en un espacio/prisión en el que los fantasmas de los anteriores inquilinos quedan atrapados, incapaces de dar el salto a lo sagrado, mientras que en el segundo es la propia casa la que asesina de manera violenta.

A la luz de esto bien podríamos preguntarnos: ¿por qué precisamente el cine de terror -e Inquilinos y Domonic son, sin duda, buenos ejemplos- hacen de la inversión de la habitabilidad heideggeriana su propia razón de existir? ¿Cómo entendemos que lo que está en juego en estos cortometrajes sea, precisamente, la "mala muerte" o la imposible aceptación de lo sagrado/descanso como umbral?

Para responder a esta pregunta no basta con apoyarse en las críticas habituales del concepto heideggeriano (Rodríguez Serrano, 2015), sino que es necesario remitir a las conexiones propuestas por Barry Curtis entre crisis económica y el cine de casas encantadas (2008: 79, 118). Como el teórico americano defiende, la destrucción del espacio habitable sirve como metáfora y resonador de las más profundas crisis en los marcos ideológicos y económicos, una suerte de desplazamiento de la angustia que emerge ante la quiebra de las certezas y la seguridad del sentido de los mecanismos de las sociedades postindustriales. Cuando los parámetros básicos de nuestra existencia social se alteran -la estabilidad laboral, la existencia de mecanismos económicos de ayuda para aquellos sujetos en riesgo de exclusión, la fiabilidad en la propia supervivencia que implica un buen sistema de sanidad o educación públicos...-, el cine de terror recoge este zeitgeist de pánico e incertidumbre y lo traduce en la casa/mundo en la que acecha lo reprimido. Esto explicaría, por ejemplo, por qué desde el comienzo de la crisis económica se han multiplicado la cantidad y calidad de los productos basados en casas encantadas. En el contexto español, esta hipótesis tiene todavía más fuerza en tanto sabemos que el propio surgimiento de la crisis económica tuvo lugar al hilo de la llamada "burbuja inmobiliaria". La corrupción, la especulación y, sobre todo, sus efectos sobre los pequeños bolsillos, se han ido asentando en una parte importante de las producciones audiovisuales patrias, llegando incluso a cristalizar en el propio género de 
terror. En el caso de Cinergía, podemos sondear claramente esta idea mediante el análisis de los protagonistas de ambos cortometrajes.

En el primero de ellos, Inquilinos, nos encontramos con el arranque típico del género: una pareja de jóvenes sin hijos (Fele Martinez y Manuela Velasco) acaban de mudarse a un piso y están acometiendo reformas. Las anteriores dueñas del piso, dos ancianas, murieron tras desplomarse el ascensor de la finca, y ahora han quedado "atrapadas" entre ambos mundos, generando sospechosos efectos paranormales. El problema del habitar es, por lo tanto, un problema de horizontes: el problema de las expectativas quebradas, del tiempo que no llega. Un problema heideggeriano en tanto no se ha hecho espacio para la "buena muerte", y un problema derrideano en tanto no hay tiempo posible para el Acontecimiento que redima (E. Vázquez, 2015). Allí donde debería empezar un cierto futuro se atora la presencia de algo antiguo, la deuda, el gesto que colapsa el devenir mismo de lo que llega. A nivel formal, esto queda exquisitamente planteado en algunos de los primeros planos del cortometraje.

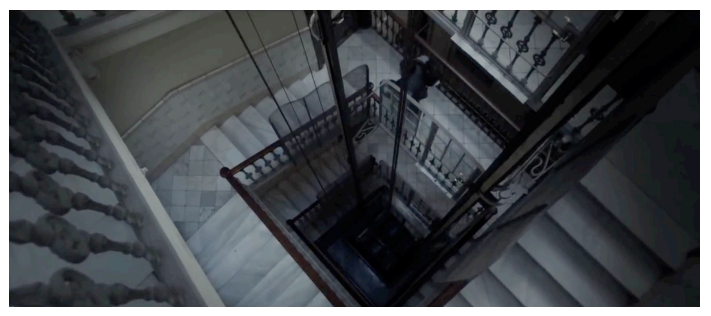

Fig. 01: Inquilinos (Jaume Balagueró, 2015)

El espacio de lo doméstico, la casa, es presentada siempre mediante planos interiores que remiten a una presencia fantasmal. Las líneas de composición aparecen voluntariamente torsionadas, ya sea mediante el subrayado de las diagonales o mediante una modificación voluntaria de la posición del espectador. Así, nos encontramos con una cámara situada al nivel de suelo, como si el dispositivo reptara o acechara, mientras que en la Fig. 01 podemos apreciar un muy violento contrapicado que pone en el centro del encuadre ese agujero negro que coincide con el lugar de la muerte de las ancianas. Ya sea mediante un plano subjetivo que se pierde en la claraboya superior del edificio o mediante los distintos juegos de montaje, toda la disposición espacial se ordena alrededor del hueco del ascensor como espacio de lo siniestro, de lo un-heimlich, de la falta: espacio donde lo doméstico se ha convertido en amenaza, en superficie inhabitable, en recordatorio constante de que los habitantes pueden morir en su propio domicilio. 
La pareja de recién casados intenta, por todos los medios, borrar las huellas de esa existencia anterior: pintar la casa, cambiar la instalación eléctrica, crear un nuevo horizonte de acontecimientos para su propia subjetividad. Sin embargo, el proceso está atorado por ese núcleo fantasmal, esa suerte de exceso de significación que emerge una y otra vez mediante las apariciones y las fotografías de las antiguas propietarias que todavía permanecen en el espacio.

Algo similar ocurre en Domonic, con la salvedad de que en esta ocasión la casa no aparece inicialmente localizada como espacio para la familia (o para la joven pareja que, como ocurría en el espot Happiness, convive felizmente en una construcción simbólica limpia de problemas y de aristas), sino como espacio del goce económico. Al igual que ocurría en el cortometraje anterior, el director incorpora casi al comienzo una serie de planos que contextualizan el hogar en el que se desarrolla la acción.

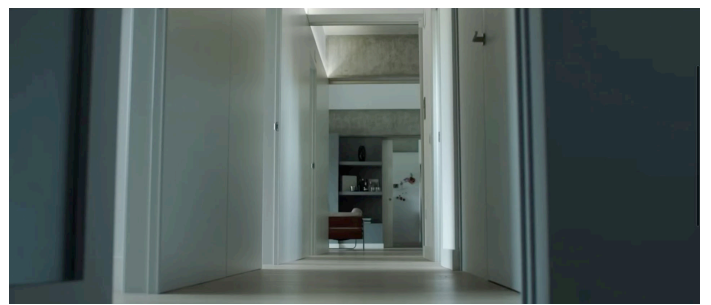

Fig. 02: Domonic (Juan Cruz, 2015)

Si Balagueró se valía de planos especialmente torsionados para generar esa idea de amenaza, hay en la propuesta de Cruz una cierta frialdad de la puesta en escena que parece valerse de las líneas horizontales y verticales propias de la casa para generar una armonía compositiva sólo muy ligeramente escorada. La construcción escenográfica remite al diseño y al lujo, como puede apreciarse por el propio diseño moderno de la vivienda (Fig. 02), en el que se remite de manera explícita a los proyectos más selectos de Le Corbusier. Los interiores, a su vez, están dominados por luces gélidas y superficies limpias que generan una suerte de soledad, de frialdad, subrayada además por la ausencia de cualquier figura humana. Más que una casa en la que se impriman las huellas del habitar podríamos encontrarnos ante una suerte de catálogo inmobiliario en el que, como ocurría con la Haus Wittgenstein, el propio programa de lo arquitectónico fagocita a lo eminentemente humano (Arenas, 2011). Algo así queda reforzado también por la conducta del protagonista (José 
Coronado), que señorea de manera estúpida en su interior, encerrado en la propia idiotez de su goce (Lacan, 1984: 62). Volviendo a la clasificación hedeggeriana, la casa de Domonic no puede hacerse cargo ni de la mortalidad de su morador -razón por la cual, muy lógicamente, no vea ningún problema en asesinarle-, ni de su relación con los elementos estrictamente naturales (los recursos energéticos) o simplemente sagrados (el recuerdo de su madre fallecida). La paradoja expuesta por el cortometraje podría ser resumida en la siguiente fórmula: ¿quién es más inhumano: aquel que vive de espaldas a su propia humanidad o el sistema informático que es capaz de sentir indignación ante el obsceno ejercicio de un goce egoísta?

La pregunta, esbozada de manera sibilina en la campaña publicitaria de una compañía que se posiciona contra los avances de las energías renovables en España, no deja de tener sus dobleces. Cruz, para matizar la posible lectura subversiva del texto, incorpora una figura materna, castradora, una suerte de trasunto edípico del HAL-9000 kubrickiano o de los autómatas de Hoffmann -figuras que, por cierto, estuvieron en el origen mismo del término unheimlich. Hay, sin embargo, un ruido textual sordo que invade todas las posibles interpretaciones del texto: el deseo de una venganza contra aquellos que han gozado demasiado, y que al hacerlo, nos han conducido a la presente situación de desamparo económico e ideológico. El espectador de Domonic, y siguiendo de nuevo una lógica esbozada por Zizek en algunos de sus trabajos vinculados con la crisis económica (2011 y 2013), puede encontrar una justicia poética innegable en la mostración de un acto violento.

Irónicamente, el más violento de los cuatro cortometrajes es el menos desarrollado narrativamente. 1:58 recrea los últimos minutos de vida en lo que parece la alucinada persecución de una mujer en la que se intuyen rasgos de enfermedad mental. Como lamentablemente ocurre tan a menudo en el cine de terror, Rodrigo Cortés propone el acercamiento a lo real del síntoma mucho más cerca de los tópicos y de los estigmas habituales (Muñoz \& Uriarte, 2006), que desde la reflexión profunda sobre las consecuencias de la mostración audiovisual de la locura.

Al igual que los dos cortos anteriores desarrollaban la violencia en torno a los parámetros de lo reprimido y lo ideológico en el habitar, aquí nos encontramos con una mostración construida únicamente en tanto lo real aparece como pulsión descontrolada, salvaje, absolutamente incapaz de ser frenada. 
En esta ocasión, el espacio doméstico es sustituido por el coche que utiliza la protagonista (Manuela Vellés) para desplazarse a toda velocidad por una carretera secundaria. Si en Domonic el retorno de la madre muerta resulta el disparadero del goce, en 1:58 es la figura del padre ausente la que domina toda la crisis psicótica de la protagonista. Valiéndose de las alucinaciones auditivas típicas del brote psicótico (Cullberg, 2007), el director incorpora una conversación inicial en la que rasgos de identidad y familiares quedan confundidos. De igual manera que Balagueró hacía bascular la puesta en escena de lo siniestro en torno al punto más angustioso del espacio (el hueco del ascensor), aquí Cortés realiza un ejercicio similar tomando como punto de referencia el rostro de la protagonista para realizar un travelling de presentación de 180 grados.

Se genera así una interesante distancia inicial con el espectador, en tanto el universo lleno de dolor y ausente de lógica (de sentido) de la protagonista queda, de alguna manera, aislado por la propia estructura del coche. El uso dominante del rojo en la iluminación y el vaho que empaña los cristales impregnan la imagen de una voluntaria artificialidad que sirve como metáfora de ese interior ardiente y doloroso por el que la protagonista bucea antes de sumergirse en su crisis de identidad. Únicamente cuando estalla el brote en toda su crudeza -cuando la protagonista musita un doloroso "Loca de los cojones", sin duda para referirse a sí misma-, el director rompe dicha barrera mediante primero un plano subjetivo, y posteriormente, un plano ya claramente tomado desde el interior del coche (Fig. 3).

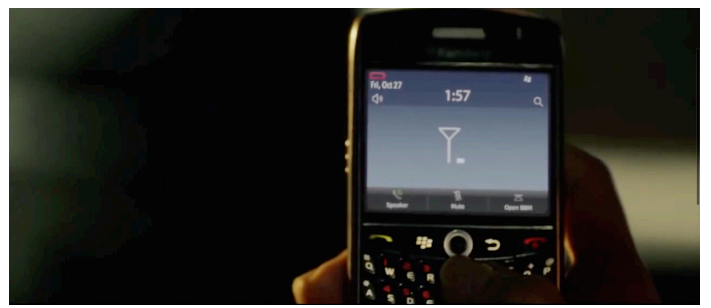

Fig. 03: 1:58 (Rodrigo Cortés, 2015)

La ruptura de esta frontera textual indica con precisión que el enunciador está a punto de desplomarse en esa misma locura que anuncia la protagonista. Y, en efecto, no sólo podremos contemplar cómo el montaje se acelera y se convierte en un elemento agresivo para con el propio universo del espectador, sino cómo los planos se van convirtiendo cada vez en elementos plásticos más inverosímiles, muchos de ellos rodados desde tiros de cámara manifiestamente antinaturalistas. Del mismo modo, la banda sonora se inunda de los 
comentarios irónicos -presumiblemente, nuevas alucinaciones auditivas- que generan una suerte de conexión de auricularización en primer grado (Jost \& Gaudreault, 1995: 144-146). De cara a nuestro estudio, lo que nos interesa señalar aquí es la mostración de la fragilidad del espacio protector - del espacio habitable- frente a la presencia del mundo exterior. De igual modo que las casas de Balagueró y Cruz eran literalmente invadidas por presencias no deseadas que bloqueaban el correcto desarrollo de lo doméstico, aquí es el coche de la protagonista el que sufre la agresión de un desconocido que la persigue y golpea hasta matarla. En los tres casos se ponía en imagen la sensación de indefensión frente a lo real, de pánico ante el florecimiento de aquella idea mayúscula que Freud rubricó con total precisión en El malestar en la cultura y de la que aquí queremos hacernos cargo:

Debido a esta primordial hostilidad entre los hombres, la sociedad civilizada se ve constantemente al borde de la desintegración. El interés que ofrece la comunidad de trabajo no bastaría para mantener su cohesión, pues las pasiones instintivas son más poderosas que los intereses racionales. (Freud, 2012: 3046).

Ciertamente, las crisis económicas e ideológicas ponen de manifiesto la frontera de la desintegración, la idea de que los logros políticos y económicos no están dados por sí mismos ni son conquistas eternas, ajenas a la propia naturaleza superviviente del ser humano. De hecho, la cita de Freud nos marca el camino para analizar el último cortometraje del proyecto: la fuerza de las pasiones por encima de los elementos racionales.

Por último, analizaremos el cortometraje Ultravioleta. Es, quizá, el que se enfrenta con mayor precisión a la problemática global que hemos intentado transmitir en el presente artículo y de ahí que su análisis resulte una suerte de integración de las diferentes hipótesis propuestas. Paco Plaza, con una precisión que resulta asombrosa, se vale de la propia formulación del branded content para poner en crisis toda la tramoya sobre la que se desenvuelve el proyecto Cinergía.

El planteamiento narrativo es el siguiente: Lola (Maribel Verdú), restauradora de arte, está arrancando un velo protector sobre una hipotética pintura perdida de William Blake. Debido a la presión de su jefa y de los inversores extranjeros de la exposición, se ve obligada a acelerar el proceso, liberando así al demonio que habitaba el interior del lienzo y siendo arrastrada hacia el cuadro.

Tras lo que parece una revisión a medio camino entre las huellas estéticas de Dario Argento -con sus correspondientes resonancias deleuzianas (Powell, 2006 y 2012)-, y las propuestas de terror español firmadas por Narciso Ibáñez Serrador en los sesenta y los setenta, se 
encuentra una metáfora brutal sobre el control de las industrias culturales por parte de las grandes compañías que lo patrocinan. Así, el corto se abre con el propio crédito que lo identifica, sobre el que se escucha la voz de la guía japonesa.

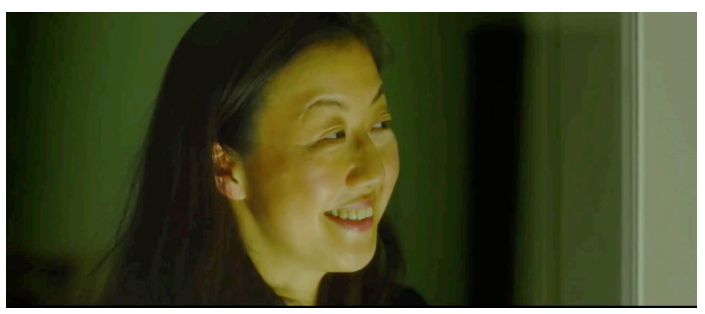

Fig. 04: Ultravioleta (Paco Plaza, 2015)

Literalmente, la primera frase de la pieza es: Esta será la joya de la colección, gracias a su generoso patrocinio, Sr. Teshigahara. Curioso que la frase se encabalgue sobre el propio espacio de la enunciación en el que la escritura se nombra a sí misma. Sin embargo, ni el Sr. Teshigahara ni su equipo accederán al verdadero territorio del arte: contemplan el lienzo parapetado tras un grueso cristal, y de hecho, sólo se comunican con Lola -un trasunto del propio director- a través de un sistema de intercomunicadores.

Dicho de otra manera: la desconexión textual entre aquellos que patrocinan y aquellos que desvelan el arte es total. Esa es, después de todo, la función textual de Lola en el cortometraje: desvelar, poner al descubierto, traer al presente, hacer que emerja aquello que permanecía escondido en el interior del cuadro: desencadenar las fuerzas demoníacas mediante un derroche desmesurado de su talento y su energía. Y la función del desvelamiento está escrita de manera literal en la pieza: no en vano, la exposición en la que trabaja Lola se Ilama, muy precisamente, Levantar el velo.

Una vez más, es necesario remitirse al aparataje estético heideggeriano para entender la profundidad de la acusación levantada por Plaza en su cortometraje. En los distintos textos que componen su teoría artística (Heidegger, 1995), el filósofo alemán se apoya explícitamente sobre el desvelamiento como responsabilidad última del artista: traer al ser entre nosotros, hacerle sitio, mostrar aquello que pertenece al ser y evitar, con ello, el ruido y el bloqueo del pensamiento que azota las sociedades postindustriales. El arte no puede rendir cuentas a nada que no sea su compromiso íntimo con la pregunta por el ser, pregunta que Plaza conecta aquí directamente con las fuerzas demoníacas encarnadas en la obra de William Blake. No se trata, por cierto, de una conexión baladí -el propio Heidegger (1958 y 1990), como es sabido, invirtió gran parte de su teoría estética alrededor de otra figura del 
romanticismo, Friedrich Hölderlin-, en tanto hay en la propia trayectoria de tres de los directores del proyecto Cinergía evidentes conexiones con el tratamiento de lo unheimlich y con una serie de cuestiones teológicas y simbólicas que los conectan con toda propiedad con el universo del pintor y poeta inglés.

Ahora bien, aquello que queda desvelado en el cuadro, aquello que no puede ser controlado ni por los patrocinadores, ni tan siquiera por la mano de los artistas que lo desvelan, es muy precisamente el núcleo terrible de la angustia, del mal-estar, la foto de la circunstancia social en la que nos encontramos. La propuesta termina, muy precisamente, con el descubrimiento de que Lola ha sido atrapada por su propio desvelamiento, convirtiéndose así en presa de su propia escritura.

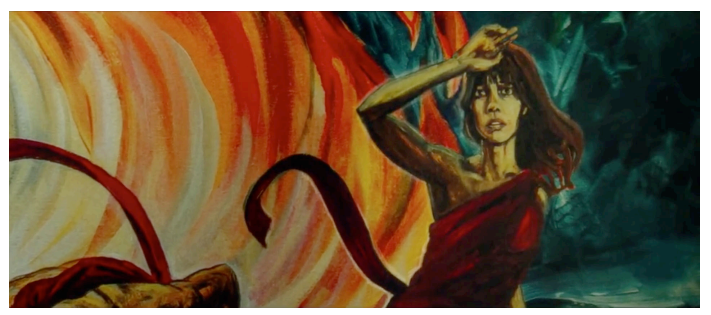

Fig. 05: Ultravioleta (Paco Plaza, 2015)

En la Figura 5 podemos ver cómo el plano queda dividido en dos secciones claras: de un lado, ese fuego infernal que coincide, muy precisamente, con esa energía que pretende vender Gas Natural Fenosa. A la derecha, la protagonista, ya atrapada tras caer presa de su propio ejercicio de desvelamiento, se ha convertido en pulpa artística, en materia pictórica pura. Sus ojos se clavan sobre el objetivo de la cámara, y Plaza refleja dicho trayecto generando un movimiento lateral de aproximación centrado en los ojos de la mujer. Un fundido a negro nos lleva al logo de la compañía y, sobre él, se escuchan los dos primeros versos de una canción de Carlos Ann: "He perdido toda la voz, regalado mi convicción, conocido el sinsabor".

Cada uno de los tres versos coincide en montaje con el último plano, el logo de la compañía y un rótulo que afirma "Dirigido por Paco Plaza". Resulta inevitable no pensar que hay una cierta voluntad irónica entre la cadena de significación con respecto a las ideas de patrocinio, branded content y creación artística. 


\section{Conclusiones}

A lo largo del presente trabajo hemos pretendido realizar un análisis fílmico más o menos exhaustivo que nos permitiera poner de manifiesto las fricciones que pueden existir entre los intereses del posicionamiento de marca y las creaciones artísticas que se generan en torno a estrategias de Branded Content. Para ello, hemos seguido el siguiente itinerario:

1) Exponer las relaciones previas existentes entre la marca Gas Natural Fenosa y el mundo de la exhibición y distribución cinematográfica, demostrando que giraban en torno a la idea del habitar.

2) Exponer cómo dicha relación utilizaba algunas ideas en torno a la crisis económica de manera resbaladiza o contradictoria -mediante posicionamientos de un optimismo políticamente correcto sin conexión alguna con la actividad real de la marca- en la campaña previa Happiness.

3) Analizar cómo los cuatro cortometrajes que componen la primera temporada de la propuesta Cinergía se hacen cargo realmente del problema del habitar en el marco de la crisis económica mundial, utilizando al menos tres estrategias distintas: la crisis del habitar doméstico (mediante la mostración de espacios familiares o íntimos inhabitables), la crisis del habitar subjetivo en lo real (mediante la mostración de quiebras personales) y la crisis de la propia función artística, dominada pero no comprendida por actividades de patrocinio y mecenazgo que resultan, a la postre, incompatibles con el potencial emancipador y autónomo del texto artístico.

\section{REFERENCIAS BIBLIOGRÁFICAS}

ARCONADA MELERO, M. Á. (1998). Hacia un nuevo contexto para mirar los espots. La publicidad en familia. Comunicar, 10, 83-95.

ARENAS, L. (2011). Fantasmas de la vida moderna. Ampliaciones y quiebras de lo subjetivo en la ciudad contemporánea. Madrid: Trotta.

CHA, J. (2013). Do online video platforms cannibalize television? How viewers are moving from old screens to new ones. Journal of Advertising Research, 53(1), 71-82. http://doi.org/10.2501/JAR-53-1-071-082

COLOMINA, B. (2010). Privacidad y publicidad: La arquitectura moderna como medio de comunicación de masas. Murcia: Colegio Oficial del Colegio de Arquitectos de Murcia.

CULLBERG, J. (2007). Psicosis : una perspectiva integradora. Madrid: Fundación para la investigación y el tratamiento de la Esquizofrenia y otros transtornos. 
CURTIS, B. (2008). Dark places: The haunted house in film. London: Reaktion Books.

E. VÁZQUEZ, M. (2015). Arquitectura de interior. Valencia: Universidad Politécnica de Valencia.

ELENA, A. (1999). Los cines periféricos. África, Oriente Medio, India. Barcelona: Paidós.

FREUD, S. (1979). Lo ominoso. En Obras completas, Tomo XVII (pp. 219-252). Buenos Aires: Amorrortu.

FREUD, S. (2012). Obras completas. Madrid: Biblioteca Nueva.

FRIEDBERG, A. (1993). Window shopping. Cinema and the postmodern. California: University of California Press.

GONZÁlEZ REQUENA, J. (2006). Clásico, manierista, postclásico. Los modos del relato en el cine de Hollywood. Valladolid: Castilla Ediciones.

GONZÁleZ SÁNCHEZ, J. F., \& GUTIÉRREZ SAN MIGUEL, B. (2013). El concepto de transversalidad en la enunciación del género cinematográfico. Revista Ámbitos, 22, 110.

HeIdegger, M. (1958). Arte y poesía. México : Fondo de Cultura Económica.

HEIDEGGER, M. (1990). De camino al habla. Barcelona : Ediciones del Serbal.

HEIDEGGER, M. (1994). Construir, habitar, pensar. In Conferencias y artículos (pp. 127142). Barcelona: Ediciones del Serbal.

HEIDEGgER, M. (1995). Caminos de bosque. Madrid : Alianza Editorial.

HEIDEGGER, M. (2009). Ser y tiempo. Madrid : Trotta.

JOST, A., \& GAUDREAULT, F. (1995). El relato cinematográfico: Ciencia y narratologia. Barcelona: Paidós.

LACAN, J. (1984). El Seminario 3: Las psicosis. Buenos Aires: Paidós.

LÉVINAS, E. (2012). Totalidad e infinito : ensayo sobre la exterioridad. Salamanca : Sígueme.

MUÑOZ, A., \& URIARTE, J. (2006). Estigma y enfermedad mental. Norte de Salud Mental, (26), 49-59. Retrieved from http://revistanorte.es/index.php/revista/article/view/438

OLIVARES MERINO, J. Á. (2011). Jaume Balagueró: En nombre de la oscuridad. Madrid: Akal.

POWELL, A. (2006). Deleuze and Horror Film. Edinburgh: Edinburgh University Press.

POWELL, A. (2012). A touch of terror: Dario Argento and Deleuze's cinematic sensorium. In European nightmares: Horror cinema in Europe since 1945 (pp. 167-177).

RODRÍGUEZ SERRANO, A. (2015). Construir, habitar, pensar, exterminar. Heidegger y la arquitectura de Auschwitz. REIA: Revista Europea de Investigación En Arquitectura, 3, $153-164$. 
SABATÉS, L. (2012). Cinesa: Cine y notoriedad. Control: La Publicidad Desde 1962, 590, 4648.

SOBA, M., \& AYDIN, M. (2013). Product Placement Efficiency in Marketing Communication Strategy. International Journal of Business and Management, 8(12), 111-116. http://doi.org/10.5539/ijbm.v8n12p111

ŽIŽEK, S. (2009). Sobre la violencia. Seis reflexiones marginales. Barcelona: Paidós.

ŽIŽEK, S. (2011). Primero como tragedia, después como farsa. Madrid: Akal.

ŽIŽEK, S. (2013). El Año que soñamos peligrosamente. Madrid : Akal.

ZUMALDE, I. (2011). La Experiencia fílmica : cine, pensamiento y emoción. Madrid : Cátedra.

\section{Agradecimientos}

El presente trabajo ha sido realizado en el marco del Proyecto de Investigación «La crisis de lo real: la representación documental e informativa en el entorno de la crisis financiera global» (P1-1A2014-05), financiado por la Universitat Jaume I, a través de la convocatoria competitiva de proyectos de investigación de la UJI (evaluados en 2014 por la Agència per a la Qualitat del Sistema Universitari de Catalunya, AQU), para el periodo 2014-2017, bajo la dirección de Javier Marzal Felici.

[Recibido: 27 de noviembre de 2015. Aceptado: 8 de febrero de 2016] 